Itinéraires Itinéraires

Littérature, textes, cultures

\title{
Narration et montage cinématographique : As I Lay Dying, de W. Faulkner
}

Aurore Touya

\section{OpenEdition}

Journals

Édition électronique

URL : http://journals.openedition.org/itineraires/2117

DOI : $10.4000 /$ itineraires. 2117

ISSN : 2427-920X

Éditeur

Pléiade

\section{Édition imprimée}

Date de publication : 1 mai 2010

Pagination : 143-155

ISBN : 978-2-296-11224-7

ISSN : 2100-1340

\section{Référence électronique}

Aurore Touya, « Narration et montage cinématographique : As I Lay Dying, de W. Faulkner », Itinéraires [En ligne], 2010-1 | 2010, mis en ligne le 01 mai 2010, consulté le 19 avril 2019. URL : http:// journals.openedition.org/itineraires/2117 ; DOI : 10.4000/itineraires.2117

\section{(C) $(\oplus \Theta$}

Itinéraires est mis à disposition selon les termes de la licence Creative Commons Attribution - Pas d'Utilisation Commerciale - Pas de Modification 4.0 International. 


\title{
Narration et montage cinématographique : As I Lay Dying, de W. Faulkner
}

\begin{abstract}
This article investigates the relationship between Faulkner's narrative technique in his polyphonic novel As I Lay Dying (1930) and the tools that filmmakers and directors can use through montage. Even though the novelist used to declare that he was not interested in film, this study reveals that novelistic polyphony and montage have much in common; and a comparison of the different narrative techniques emphasizes what those two forms of art share.
\end{abstract}

Keywords : Faulkner, film, structure, narrative technique, montage

Mots clés : Faulkner, cinéma, structure, narration, montage

La discipline comparatiste telle qu'elle a été conçue au début du $\mathrm{xx}^{\mathrm{e}}$ siècle permet de mettre en parallèle et de faire dialoguer les œuvres d'auteurs différents mais liés par un aspect singulier de leur travail. Or depuis quelques dizaines d'années, cette discipline s'ouvre à des comparaisons plus larges, et peut désormais rapprocher différentes formes d'art de la littérature.

Cette ouverture de la discipline comparatiste à la peinture, à la photographie ou au cinéma par exemple, ne signifie pas pour autant une perte d'intérêt pour le texte littéraire; au contraire, le détour par une autre forme d'art est parfois nécessaire et peut permettre de renouveler le champ de la recherche en littérature, en faisant surgir de nouvelles questions.

C'est ce qui s'observe en tout cas pour la littérature américaine du $\mathrm{XX}^{\mathrm{e}}$ siècle, souvent rapprochée des pratiques cinématographiques qui lui furent contemporaines : les grands romanciers américains ont pu être comparés aux scénaristes et réalisateurs de leur temps. De plus, Steinbeck, Dos Passos ou Hemingway, parmi d'autres, s'intéressaient au cinéma en tant qu'art du récit, et certains de leurs textes ont été portés à l'écran. 
Il semble cependant en aller autrement pour William Faulkner. Si le romancier a travaillé comme scénariste à Hollywood à partir de 1932, cela fut toujours à des fins financières, afin de pouvoir solder des dettes qu'il avait contractées et se consacrer ensuite pleinement à l'écriture de ses romans : Faulkner écrivait essentiellement des dialogues pour des scénarios dont il se désintéressait. De même, si certains de ses romans furent l'objet d'adaptations ${ }^{1}$, Faulkner n'était pas un cinéphile et ne manifestait guère d'intérêt pour les expérimentations cinématographiques pratiquées par ses contemporains avec qui il aurait pu aisément s'entretenir à Hollywood.

Et pourtant, en rapprochant le cinéma tel qu'il existe au début des années 1930 des romans écrits alors par Faulkner, certaines ressemblances apparaissent. Il semblerait que l'écriture faulknérienne et l'écriture cinématographique soient toutes deux marquées du sceau du modernisme, ce courant de pensée qui traverse les différents champs de la création artistique du début du $\mathrm{Xx}^{\mathrm{e}}$ siècle. Ce rapprochement continue ainsi d'occuper les spécialistes de l'œuvre de Faulkner, et il sera d'ailleurs en 2010 au centre du colloque annuel organisé par l'université du Mississippi ${ }^{2}$. Les questions soulevées par cette comparaison entre les deux arts sont en effet nombreuses et complexes : que ce soit du point de vue biographique de la présence de Faulkner à Hollywood, de celui des rapports de l'auteur à la culture populaire mise en œuvre à travers le cinéma, ou des comparaisons établies entre littérature et cinéma - ces deux arts qui se consacrent à raconter des histoires - de nombreuses interrogations demeurent.

Car il semble bien que certains des romans de Faulkner aient des choses en commun avec ce qui se joue au même moment au cinéma. En effet, les premiers romans de Faulkner, notamment The Sound and The Fury (1929) et As I Lay Dying (1930), respectivement traduits en français sous les titres Le Bruit et la Fureur et Tandis que j'agonise, sont de véritables champs d'exploration et d'expérimentation de nouvelles techniques narratives, au moment même où le cinéma moderniste lance lui aussi ce type d'expériences. Traversés par la pensée moderniste, et par l'importance qu'elle accorde à la forme autant qu'au fond - si ce n'est plus - cinéma et littérature soulèvent des questions proches : comment raconter une histoire? Quelles distorsions le récit peut-il ou doit-il subir, afin de renforcer la portée et le sens de l'histoire mise en scène? Comment organiser le récit, en préservant l'intelligibilité de la suite des événements mais sans recourir forcément à la narration linéaire canonique qui épouse l'enchaînement chronologique des faits?

1. Par exemple, Sanctuary (1931) est adapté au cinéma par Stephen Roberts et devient The Story of Temple Drake, version qui édulcore grandement la violence du roman, et fait disparaître la fameuse scène de l'épi de maïs.

2. L'édition 2010 de « The Faulkner and Yoknapatawpha Conference », colloque international organisé depuis trente-sept ans par l'université du Mississippi Ole Miss à Oxford, MS, a pour titre « Faulkner \& Film ». 
Ces questions se posent, de façon différente certes, mais équivalente, pour chacun de ces deux arts. Et si l'on observe plus précisément le début de l'œuvre de Faulkner, on s'aperçoit qu'elles se posent avec une acuité particulière pour As I Lay Dying, qui pousse plus avant les expérimentations déjà à l'œuvre dans The Sound and The Fury, publié un an auparavant.

C'est pourquoi nous étudierons ici les analogies qui existent entre le découpage narratif que Faulkner expérimente en 1930 dans As I Lay Dying et les possibilités d'organisation des scènes d'un film liées aux pratiques d'écriture et de montage telles qu'elles existent au cinéma. Il s'agit de voir, en abordant de manière comparatiste la construction et l'esthétique de l'œuvre, comment certains modes de découpage narratif propres au roman dans As I Lay Dying peuvent être rapprochés de ce qui se fait au cinéma, et comment les différentes techniques de la narration et du montage soulignent les enjeux communs de ces deux supports de fiction que sont la littérature et le cinéma - à savoir raconter une histoire, présenter une vision du monde, délivrer une interprétation du sens de l'existence humaine.

\section{Narration polyphonique et montage cinématographique : enjeux}

\section{Des intérêts communs}

Le roman de Faulkner et le cinéma des années 1930 partagent un intérêt pour des questions d'organisation et de découpage du récit. Ces deux formes d'art sont unies par l'importance que revêt, pour le Modernism, la forme de l'objet littéraire ou cinématographique : ce qui compte, ce n'est plus tant l'histoire que le roman ou le film raconte, que la façon dont elle y est racontée. Devant les changements de ce début de siècle, les artistes s'interrogent en effet sur la technique : et si de nouveaux outils étaient à trouver dans l'art, pour le renouveler? Quels nouveaux modes de perception faut-il proposer, afin de traduire artistiquement ces problématiques?

À chaque forme d'art son outil : le roman est régi par une narration, traditionnellement menée par un narrateur extérieur, omniscient, capable de pénétrer les esprits et de rapporter les pensées et les propos des personnages. Le cinéma quant à lui, qui se dote du son à partir des années 1920 et renforce ainsi ses possibilités d'expression, possède sa syntaxe propre : il s'agit du montage, qui vient compléter, après le tournage, l'organisation du film prévue lors de l'écriture du scénario.

Le montage permet la construction du film, par le découpage, l'agencement et la mise en forme des images. Les scènes filmées à partir du scénario ne sont que le matériau du cinéma, le premier support qui est nécessairement remanié, découpé et organisé selon un ordre qui a été prétabli au moment de l'écriture du scénario et de son organisation en scènes, mais qui peut être jusqu'au dernier moment modifié. C'est dans un second temps, 
dans les studios de montage et à l'aide de techniciens spécialisés, que le film trouve sa forme définitive. Il s'écrit une seconde fois, dans la construction après-coup grâce au montage, qui lui confère son rythme propre, sa structure, sa progression, et lui donne ainsi sa singularité véritable.

\section{As I Lay Dying : un roman d'une structure singulière}

Dans un entretien accordé à Frederick Gwynn et Joseph Blotner, William Faulkner déclara au sujet de sa méthode d'écriture romanesque :

\footnotetext{
Unless a book follows a simple direct line such as a story of adventure, it becomes a series of pieces. It's a good deal like dressing a showcase window. It takes a certain amount of judgment and taste to arrange the different pieces in the most effective place in juxtaposition to one another ${ }^{3}$.
}

Et en effet, comme la vitrine d'un magasin fascinant, le roman faulknérien donne à admirer les objets les plus inattendus les uns à côté des autres, dans une juxtaposition tout d'abord déconcertante, mais qui dévoile peu à peu son sens et sa légitimité.

C'est le cas avec le deuxième roman de Faulkner. Publié en 1930, un an après The Sound \& The Fury, As I Lay Dying - traduit en français dès 1934 par Maurice-Edgar Coindreau, sous le titre Tandis que j'agonise, chez Gallimard - poursuit les expérimentations structurelles lancées par le roman précédent. En effet, The Sound \& The Fury proposait d'écouter quatre voix, quatre versions d'une même histoire de famille, tourbillonnant autour du décès de la grand-mère des enfants Compson dans le Mississippi des années 1920. Ce sont successivement Benjy, l'idiot de la famille, son frère Quentin, et son autre frère Jason qui relatent chacun à sa façon, de son point de vue et à des dates différentes - au moyen du pronom personnel de première personne du singulier, $I$ - cette histoire et les événements qu'elle fait remonter dans leur mémoire. La dernière partie est menée par un narrateur extérieur, qui épouse la perspective de Dilsey, la servante noire qui maintient ensemble les débris restants de la famille Compson après le suicide de Quentin et la fuite de sa sœur Caddy.

La diégèse de As I Lay Dying tourne elle aussi autour de la disparition d'un des personnages : au début du roman, Addie Bundren, mère de famille et épouse d'Anse Bundren, est à l'agonie dans une des chambres de la maison familiale. Après son décès, son mari et ses enfants se mettent en route pour Jefferson, dans le Mississippi, avec le cercueil renfermant le

3. «Sauf lorsqu'un livre suit une simple ligne directe comme dans le cas d'un roman d'aventure, il devient une série d'objets. C'est comme organiser une vitrine. Cela demande une certaine dose de bon sens et de talent pour disposer les différents objets à l'endroit le plus judicieux, les uns à côté des autres ", traduction personnelle. Entretien cité par Blair Labatt dans Faulkner The Storyteller, Tuscaloosak, University of Alabama Press, 2005. 
corps d'Addie, afin de l'enterrer près des siens comme elle l'a demandé de son vivant. Mais le voyage s'avère périlleux, du fait tant de certains événements inattendus que des intérêts privés de chacun des personnages qui menacent le groupe.

As I Lay Dying redouble ainsi la fragmentation du récit: de quatre sections dans The Sound \& The Fury, on passe à quarante-neuf. Ces sections apparaissent lors de la première lecture comme des monologues intérieurs : chaque section est consacrée à la voix d'un personnage, annoncé en majuscules dès l'ouverture de chaque chapitre, et c'est en ce sens que le roman peut être qualifié de polyphonique.

Un classement des quinze personnages présents dans le roman, selon le nombre de sections consacrées à leur parole, permet de dégager certains points importants :

\begin{tabular}{|l|r|}
\hline Darl & 19 \\
\hline Vardaman & 10 \\
\hline Vernon Tull & 6 \\
\hline Cash & 5 \\
\hline Dewey Dell & 4 \\
\hline Cora Tull & 3 \\
\hline Anse & 3 \\
\hline Peabody & 2 \\
\hline Moseley & 1 \\
\hline Samson & 1 \\
\hline Mac Gowan & 1 \\
\hline Armstid & 1 \\
\hline Whitfield & 1 \\
\hline Jewel & 1 \\
\hline Addie & 1 \\
\hline
\end{tabular}

De toute évidence, la parole de Darl, avec ses dix-neuf sections, dépasse largement celle de tous les autres personnages présents dans le roman. Vient ensuite Vardaman, qui possède quant à lui une dizaine de sections. Il faut de plus noter que les seuls membres de la famille Bundren à ne posséder qu'une section sont Jewel et sa mère Addie. Ces deux personnages sont ainsi davantage donnés à voir par ce que les autres en disent ou pensent que par une incursion dans leur conscience. Cependant, une différence est à noter : la section consacrée à la parole de Jewel occupe la quatrième place dans le roman, tandis que celle d'Addie occupe la quarantième. L'intériorité du personnage de Jewel, intervenant en tout début de roman, précède ainsi la construction de son image par les autres personnages, tandis que celle d'Addie, intervenant tardivement, et qui plus est post mortem dans la diégèse, se place en porte-à-faux par rapport à tout ce qui a déjà été entendu à son sujet dans les autres sections. 
Mais ce n'est pas le nombre de sections consacrées à un personnage qui dit son importance : Addie et Armstid, par exemple, possèdent tous deux une seule section, mais il est évident qu'ils pèsent différemment dans l'organisation de la diégèse. Addie, le personnage à l'agonie dont le décès et les obsèques lancent la quête romanesque, est le personnage central du roman, celui autour de qui tout s'organise, tandis qu'Armstid n'est qu'un témoin, une figure permettant certes de construire un événement du récit, mais sans commune mesure avec la mort d'Addie et ce qu'elle engage pour l'organisation du roman.

Chaque section, qui peut d'un point de vue technique être soit assimilée à un monologue intérieur, soit au stream of consciousness, ou mêler les deux, en même temps qu'elle désigne un moment de l'action - celui où le personnage est traversé par ces pensées - nous livre un reflet plus ou moins déformé de la réalité dans une conscience en train de la percevoir et de la vivre. La fragmentation du roman est de ce fait poussée à l'extrême : certaines sections peuvent être très brèves, comme en témoigne la section XIX, troisième prise de parole de Vardaman et se résumant à la phrase : «My mother is a fish ${ }^{4}$ » (« Ma mère est un poisson »), ou peuvent au contraire s'étendre sur plusieurs pages, dans le cas des sections consacrées à Darl, ou de celle d'Addie. Cette segmentation semble parfois donner à voir des saynètes de théâtre, créant une forte impression de discontinuité, une rotation comme kaléidoscopique des points de vue.

Ainsi, à l'ouverture de chaque nouvelle section, la perspective se déplace, l'éclairage se modifie, prenant le lecteur à contre-pied et l'obligeant à de constants rétablissements s'il tient à suivre la narration à travers ses multiples avatars. Avec As I Lay Dying, William Faulkner se met au défi de raconter l'histoire des Bundren malgré - et à travers - toutes les brisures qu'il fait connaître à son récit.

Mais ces brisures, ces interruptions de narration, si elles semblent d'abord ralentir et complexifier l'action, relèvent bien d'un montage, d'un agencement des points de vue et des versions organisant une suite de scènes qui finissent par constituer le récit.

Voyons alors comment fonctionnent les procédés de découpage et d'agencement de ces sections, tout en les comparant avec les procédés de montage cinématographique les plus courants.

\section{Découpage textuel et montage visuel : comparaison des techniques}

Bien qu'une telle étude ne puisse être complètement menée à bien dans un cadre aussi restreint que celui de cet article, il semble nécessaire d'établir certains parallèles entre la technique narrative polyphonique que

4. William Faulkner, As I Lay Dying, New York, Random House, 1930, p. 76. Toutes les références ultérieures à ce roman seront données dans cette édition. 
Faulkner invente dans As I Lay Dying, et les procédés de narration propres au cinéma, afin de se faire une idée plus juste et plus précise de ce qui unit l'auteur au cinéma en termes narratologiques, et de voir ce que peuvent partager d'un point de vue esthétique le cinéma et la littérature de fiction.

\section{Visions de personnages, cadrage et mouvements de caméra}

Lors de la lecture du roman de Faulkner, les monologues intérieurs apparaissent comme autant de visions des personnages, qui décuplent la vision du monde que développait jusqu'alors le narrateur omniscient dans la tradition narrative romanesque. Au lieu d'une seule version des faits, ce sont ici des versions différentes et variables qui sont livrées par les personnages eux-mêmes. Or, chacune de ces versions revient à une vision : chaque section donne à lire ce que pense le personnage, en fonction de ce qu'il a vu, ou au moment même où il le voit - ce qui explique cet usage fréquent du présent de l'indicatif chez plusieurs narrateurs personnages.

L'incipit du roman, qui s'ouvre sur une section consacrée à la parole de Darl, souligne d'emblée cette importance du regard, et précise au lecteur où se trouve ce qui serait au cinéma la caméra, le point de vue d'où embrasser la scène décrite par Darl :

Jewel and I come up from the field, following the path in single file. Although I am fifteen feet ahead of him, anyone watching us from the cotton-house can see Jewel's frayed and broken straw hat a full head above my own ${ }^{5}$.

Ainsi, le point de vue - ici présenté par l'équivalent d'un mouvement de caméra panoramique, à moins que l'on n'envisage un travelling qui suivrait les deux silhouettes le long du chemin - est précisé : c'est depuis la grange à coton que la scène est vue.

Ce procédé est d'une grande habileté : comme le fait un réalisateur avec un spectateur de cinéma, le personnage narrateur indique d'entrée au lecteur où se positionner et comment regarder la scène, bien que cette dernière soit décrite depuis un autre endroit, à savoir le chemin que remonte Darl en compagnie de son frère Jewel. En indiquant la cotton-house comme point de perception, le narrateur permet de cadrer la scène - et ce faisant de planter le décor des événements à venir, respectant ainsi les attentes d'un incipit romanesque - tout en y introduisant un mouvement, celui des deux frères sur le chemin, qui lance l'action et fait affleurer les

5. «Jewel et moi remontons du champ, suivant le chemin l'un derrière l'autre. Bien que je le dépasse de quinze pieds, quiconque nous regarde depuis la grange à coton peut voir le chapeau de paille tout abîmé de Jewel à la distance d'une tête au-dessus du mien », traduction personnelle. Section I, première sur dix-neuf à être consacrée à Darl, p. 1. 
premières pensées du personnage dont nous lisons le monologue, comme une voix off qui nous fait pénétrer dans le récit.

Mais là encore, Faulkner nous réserve une surprise : le lecteur ne peut s'attacher durablement à cette voix off; il doit au contraire en écouter plusieurs successivement, et s'abandonner au tourbillon des voix qui épouse le kaléidoscope des visions.

\section{Alternance de monologues et alternance de plans}

L'alternance entre plans courts et plans longs - qui deviennent parfois des plans-séquences, des scènes entières filmées à partir d'un seul plan - est constitutive du rythme même de la narration cinématographique. Souvent conditionnée par des règles précises en fonction du résultat souhaité, elle confère au film son identité et sa dynamique de narration, lente, rapide ou saccadée.

À ce titre, As I Lay Dying propose une succession de plans rapides, voire très rapides. Les sections consacrées à chaque personnage, qui peuvent être lues comme autant de plans sur un visage, ou de caméras subjectives filmant à travers les yeux des personnages, n'excèdent jamais une dizaine de pages, et se limitent souvent à quelques lignes, voire à quelques mots - chez Cash et Vardaman en particulier. Darl est le personnage dont les monologues intérieurs sont les plus longs : au cinéma, cela correspondrait aux plans les plus longs du film; tandis que la phrase qui hante Vardaman dans les sections XIII et XIX reviendrait à un plan-éclair donnant à voir une superposition de l'image de sa mère et d'un poisson.

En optant pour des sections de taille variable, et en faisant alterner l'extrême brièveté de certains chapitres à des développements plus amples - comme le monologue post mortem d'Addie, qui occupe la quarantième section du roman - Faulkner donne à son roman un rythme cadencé, où la vision de chacun des personnages a le temps de se déployer, mais est toujours menacée d'interruption, ce qui soutient la dynamique de cette narration fragmentée, et équivaut à ce qui se pratique aujourd'hui dans les films choraux ou en étoile.

\section{Prolepse et analepse, ellipse et flash-back}

La prolepse et l'analepse permettent dans un récit de faire un saut, respectivement en avant et en arrière, dans la chronologie : elles sont les outils nécessaires au traitement du temps dans le récit, et permettent de se défaire de la linéarité de la chronologie, par des accélérations ou des retours dans le passé, qui confèrent à l'histoire son propre rythme. Précisons de plus que prolepse et analepse sont une des caractéristiques du roman polyphonique : en même temps qu'il fait varier les narrateurs, le roman polyphonique a la capacité de les faire parler depuis différents chronotopes, et de changer 
ainsi souvent d'espaces-temps, de revenir dans le passé ou d'anticiper vers l'avenir.

Or, les équivalents de la prolespe et de l'analepse au cinéma jouent un rôle crucial dans l'économie et le rythme d'un film : l'ellipse, qui passe sous silence certains événements, et le flash-back, qui revient sur un épisode passé, sont des outils incontournables pour tout film qui ne suit pas une trame exactement chronologique.

Dans As I Lay Dying, prolepse et analepse sont présentes, et servent certains événements primordiaux pour l'organisation du roman. Ainsi, c'est une prolepse qui sert au traitement de l'événement de la mort d'Addie dans la diégèse : la mort y est passée sous silence, sauf dans l'impossible récit de Darl, qui relate le dernier souffle de sa mère sans y assister physiquement. On passe directement de la vision d'Addie sur son lit, éventrée par sa fille Dewey Dell à la section XI, dont la narration est confiée au médecin de campagne Peabody, à la réaction violente de Vardaman, le plus jeune fils de la défunte, qui vient de comprendre. À part dans le monologue de Darl - le fou-devin qui imagine la scène ou y assiste à distance, sans aucune possibilité de vraisemblance romanesque - la mort d'Addie est passée sous silence, et on ne peut en lire que ses conséquences sur les autres personnages, comme la colère et le désarroi de Vardaman (sections XIII, XV et XIX), le dîner d'Anse et du docteur Peabody (section XIV), la cérémonie funèbre relatée par Tull (section XX).

Des analepses s'observent quant à elles à plusieurs reprises, comme par exemple dans la section VII, consacrée à la parole de Dewey Dell, où elle relate son aventure avec Lafe, et la réaction de Darl qui a deviné ce qui s'est passé :

He said he knew without the words like he told me that ma is going to die without words ${ }^{6}$.

Un autre exemple d'analepse s'observe également à l'échelle de l'ensemble de la section XXXII, où Darl livre le récit du spell of sleeping, de la « crise de sommeil » de son frère Jewel à quinze ans.

Ces accélérations et ces retours en arrière permettent deux choses, dans le roman comme au cinéma : ils organisent des ruptures de rythme, qui veillent à dynamiser le récit et à le faire sortir des cadres canoniques du récit chronologique; ils permettent aussi d'approfondir les connaissances du lecteur ou du spectateur, en revenant sur des événements passés capables d'éclaircir la situation - ou au contraire, comme souvent chez Faulkner, de la compliquer encore un peu plus.

6. " Il a dit qu'il savait sans les mots comme il m'a dit que maman va mourir sans mots », traduction personnelle. Section VII, première sur quatre à être consacrée à Dewey Dell, p. 23. 


\section{Concomitance des événements narrés et montage alterné}

Les vingt premières sections du roman présentent, par l'alternance des monologues des différents personnages, les activités de chacun alors qu'Addie est à l'agonie dans sa chambre de la maison familiale. Les principaux personnages y sont représentés : Darl, Jewel, Cash, Dewey Dell et Vardaman, c'est-à-dire les cinq enfants d'Addie; son époux Anse; les voisins Cora et Tull; et le médecin Peabody. Or, en faisant alterner ainsi les monologues de personnages principaux correspondant à autant de pensées concomitantes, le romancier utilise l'équivalent en littérature du montage alterné, cette pratique qui donne à voir successivement des actions simultanées : la ronde des monologues et le changement successif des perspectives d'une section à l'autre correspondent à autant de caméras qui filmeraient la vision de chacun des protagonistes à travers leurs yeux, épousant à tour de rôle la perception de chacun à un même moment de l'histoire. On peut ainsi considérer que le monologue intérieur, qui donne à lire les pensées plus ou moins désordonnées du personnage, est dans le roman polyphonique l'équivalent de la caméra subjective; et ces deux façons de donner à lire ou à voir la perspective du personnage sont déclinées les unes après les autres mais correspondent à une simultanéité dans la chronologie.

\section{Section atypique et montage parallèle}

Enfin, on peut observer une variante de la correspondance entre alternance des monologues (et ce faisant, alternance des référents désignés par le pronom personnel $I$ qui désigne le locuteur) et montage alterné : il s'agit de la proximité entre certaines sections atypiques du roman et la pratique du montage parallèle. Le montage parallèle permet en effet de faire alterner des plans ou des séquences qui ne sont liés ni par un rapport de simultanéité (le fameux "et pendant ce temps-là... ») ni par un rapport de causalité. Ce type de montage revient à associer deux images qui n'auraient pas dû se rencontrer; il correspond à l'écran à une métaphore ou à une association inattendue dans un texte.

Or cet usage associatif est à l'œuvre dans As I Lay Dying, sous la forme de deux monologues surprenants qui se suivent. Il s'agit des sections XVIII et XIX, respectivement menées par les voix de Cash et de Vardaman.

La section XVIII correspond à une liste en treize points numérotés, introduits par la laconique précision :

I made it on the bevel ${ }^{7}$

7. «Je l'ai taillé en biseau », traduction personnelle. Section XVIII, première sur cinq à être consacrée à Cash, p. 75. 
indiquant la manière dont Cash a construit le cercueil de sa mère, désigné dans la phrase par le minimaliste pronom $i t$, en taillant en biseau les angles d'une planche de bois. Cette obsession de la construction la plus adaptée revient dans plusieurs monologues de Cash, notamment à la section XXXVIII; mais ce qu'il faut ici souligner, c'est la singulière forme de liste numérotée que prend le monologue. À chaque numéro semble d'abord correspondre une remarque ou une instruction, afin de mener à bien la conception du cercueil :

1. There is more surface for the nails to grip.

2. There is twice the gripping-surface to each seam ${ }^{8}$.

Néanmoins, la démonstration tourne à l'inattendu, et certaines parties numérotées de la liste sont consacrées à des tournures réduites, qui épousent un moment de la pensée du personnage sans correspondre véritablement à l'objectif d'explication :
6. Except.
$[\ldots]$
8. Animal magnetism ${ }^{9}$.

Cette étonnante association est renforcée par l'agencement de la section suivante. C'est cette fois Vardaman, le benjamin, qui pense :

My mother is a fish ${ }^{10}$.

Passée la surprise lors de la lecture d'un tel monologue intérieur, on comprend que le personnage de Vardaman associe les événements qu'il vit - le décès de sa mère - avec des objets du quotidien, donnant lieu à d'étranges raccourcis poétiques qui reposent sur l'association imagée de deux objets selon un seul point commun. La mort de la mère correspond dans la diégèse au moment de fierté du garçon d'avoir pêché un gros poisson : les deux corps morts, celui de la mère et celui du poisson, s'associent alors dans l'esprit du jeune garçon sous la forme de cette métaphore métonymique, sans doute appréciée des surréalistes.

Ainsi, les sections XVIII et XIX ne correspondent pas aux attentes habituelles générées par la forme du monologue intérieur : elles ménagent en leur sein surprise et étonnement. Et c'est leur agencement en deux sections atypiques suivies, comme le ferait au cinéma un montage parallèle juxtaposant les images rapides et singulières du cercueil et de la mère-poisson,

8. «1. La surface d'adhérence des clous est plus grande. 2. Il y a le double de surface d'adhérence pour chaque arête », traduction personelle.

9. «6. Sauf. [...] 8. Magnétisme animal», traduction personnelle.

10. Section XIX, troisième sur dix à être consacrée à Vardaman, p. 76. 
qui renforce leur sens et donne pleinement à lire, de manière métaphorique, le trouble des fils d'Addie.

Ainsi donc, la comparaison méthodique entre certains outils littéraires et certains outils cinématographiques permet de s'interroger sur l'apport esthétique d'un tel usage de ces procédés. Ce qui semble le plus intéressant au terme de cette succincte étude comparée, c'est de voir que cette multiplicité de voix et de visions interroge la véracité d'une version de l'histoire : multiplier les versions et agencer les monologues dans le roman, juxtaposer les visions au cinéma et par exemple filmer la même scène depuis des points de vue différents, comme le font toujours aujourd'hui certains réalisateurs ${ }^{11}$, c'est souligner qu'une même histoire peut être valable de plusieurs façons. La version recevable et unique, offerte de façon linéaire et incontestable au lecteur par le narrateur extérieur omniscient, ne vaut plus : elle est contestée et finalement balayée par la multiplicité des voix du roman polyphonique et par les plans subjectifs d'une même scène que permet le montage.

En recourant à cette multiplication des versions et des récits, le romancier comme le cinéaste peuvent désormais faire entrer dans l'œuvre de fiction la possibilité du doute, la cœxistence de plusieurs vérités, et permettre à leur œuvre d'épouser la complexité du monde. En refusant de proposer une seule version des faits à l'exception des autres, ils se refusent à imposer une vision du monde qui prévaudrait sur les autres et proposent à la place, comme l'écrit Édouard Glissant, de « défaire cette vision du réel ou du vrai qui serait unicitaire [...] et [d']y introduire le multiple, l'incertain, le relatif ${ }^{12} \gg$.

En conclusion, il apparaît donc que, malgré les propos que tenait Faulkner sur Hollywood et sur le cinéma de son temps, son œuvre et en particulier ce roman datant de 1930 expérimentent des techniques narratives qui interrogent l'organisation et le sens du récit, comme peuvent le faire au cinéma le montage et l'écriture filmique.

Ce sont ainsi des questions similaires qui se posent, à peu près au même moment en ce début de $\mathrm{Xx}^{\mathrm{e}}$ siècle, à ces deux arts de la fiction que sont respectivement la littérature et le cinéma, tous deux traversés par les interrogations que soulève le mouvement moderniste sur le sens de l'art et sur la forme que peut ou doit revêtir chaque œuvre. Certes, il ne faudrait pas forcer la comparaison et faire de As I Lay Dying un roman essentiellement influencé par le traitement filmique - on a souligné la relation ambiguë de Faulkner à Hollywood, et certains aspects du roman échappent au rapprochement avec le cinéma, notamment par l'importance accordée à la

11. On peut citer les célèbres films d'Alejandro González Iñárritu, notamment Amores Perros, 2000, 21 Grams, 2003 et Babel, 2006, ou de Gus Van Sant, comme Elephant, 2003.

12. Édouard Glissant, Faulkner, Mississippi, Paris, Stock, 1996, p. 269. 
retranscription de la pensée, censée être impénétrable, par le monologue écrit. Néanmoins, il apparaît clairement que certaines questions esthétiques, soulevées dans le roman par les outils de narration et le complexe travail de découpage des voix des personnages, se posent également au cinéma des années 1930; et elles animent encore aujourd'hui la réflexion sur le septième art.

Aurore Touya

Université Paris $13-C E N E L$ 